\title{
Repurposing Human Osteoarthritic Cartilage as a Bone Graft Substitute in an Athymic Rat Posterolateral Spinal Fusion Model
}

\author{
ALAN B.C. DANG, MD, ${ }^{1,2}$ HELENA HONG, BA, ${ }^{3}$ KATIE LEE, BA, ${ }^{2}$ TAMMY LUAN, BA, ${ }^{1}$ SANJAY \\ REDDY, MS, ${ }^{1}$ ALFRED C. KUO, MD, PHD ${ }^{1,2}$ \\ ${ }^{1}$ Orthopaedic Section, Surgical Service, San Francisco VA Medical Center, San Francisco, California, ${ }^{2}$ Department of Orthopaedic Surgery, University of \\ California, San Francisco, California, ${ }^{3}$ Washington University School of Medicine, St Louis, Missouri
}

\begin{abstract}
Background: Spinal fusion involves both endochondral and intramembranous bone formation. We previously demonstrated that endochondral cartilage grafts that were derived from human osteoarthritic (OA) articular cartilage can be used as a bone graft in mouse models. We hypothesized that OA cartilage could also be recycled and repurposed as a bone graft substitute in a posterolateral lumbar spinal fusion model in athymic rats.

Methods: OA articular cartilage was obtained from the femoral resection of a healthy 60 -year-old man undergoing elective total knee arthroplasty. The chondrocytes recovered from this tissue were dedifferentiated in monolayer tissue culture and then transitioned to culture conditions that promote chondrocyte hypertrophy. The resultant cell pellets were then used as bone graft substitute for single-level posterolateral spinal fusion in 5 athymic rats. Decortication alone was used as the control group. Spinal fusion was assessed with manual palpation, micro-computed tomography, and histologic analysis.

Results: In the experimental group, micro-computed tomography at 4 and 8 weeks demonstrated bilateral fusion in 4 of 5 animals and unilateral fusion in 1 animal. At 8 weeks, manual palpation and histologic analysis showed direct correlation with the radiographic findings. Animals undergoing decortication alone failed to generate any spinal fusion. The difference in the fusion rate between groups was statistically significant with respect to both unilateral fusion $(P=$ $.047)$ and bilateral fusion $(P=.007)$.

Conclusions: In the absence of additional surgically implanted bone graft, hypertrophic chondrocyte grafts are sufficient for generating single-level posterolateral lumbar fusion in athymic rats.

Clinical Relevance: This animal study demonstrates that cartilage harvested from OA knees can be used as a bone graft substitute. Commercially available cell-based bone grafts have previously only used mesenchymal stem cells or osteoblast precursor cells.
\end{abstract}

New Technology

Keywords: endochondral bone formation, spinal fusion, animal models

\section{INTRODUCTION}

Spinal fusion is a common procedure used in the treatment of symptomatic spondylolisthesis with neurogenic claudication and/or radiculopathy, spinal instability, and spinal deformity. Despite advances in surgical technique, pseudarthrosis (failure of the fusion) still occurs in $18 \%$ of patients, ${ }^{1-5}$ and with conventional bone graft substitutes failure is as high as 30\%.6,7 With more than 413000 spinal fusions performed each year in the United States ${ }^{8}$ and an average direct and indirect cost of $\$ 41631$ for the treatment of pseudarthrosis with revision surgery, ${ }^{9}$ this accounts for as much as $\$ 5$ billion in added costs to the US health care system. Patients undergoing revision surgery face increased perioperative risks of infection, dural tears, and pain, as well as the prospect of enduring months of uncertainty before it is known whether the revision surgery was successful or not. ${ }^{10,11}$

One of the most efficacious bone graft substitutes in clinical use is rhBMP-2. It has been shown to have higher rates of fusion relative to the "gold standard" of iliac crest bone autograft in spine surgery and oromaxillofacial surgery. ${ }^{12,13}$ In select patient groups, such as those older than 60 years, the use of rhBMP-2 may even be cost-effective compared with iliac crest. ${ }^{14}$ The fundamental problem with rhBMP-2 is safety. ${ }^{15}$ Risks include 
fatal airway compromise when used in cervical spinal fusion secondary to local swelling. ${ }^{16}$ Other concerns include the formation of heterotopic bone, which can cause neurologic injury. ${ }^{17,18}$ In 2008, these risks led the US Food and Drug Administration to issue a public health notification alerting clinicians to the complications associated with offlabel rhBMP2 use in the cervical spine. ${ }^{16}$ Safer alternatives to rhBMP-2 include demineralized bone matrices as well as osteoconductive scaffolds that work as bone graft extenders to increase the volume of autologous grafts. However, scaffolds generally have not shown superiority to iliac crest autologous grafts. ${ }^{19}$ Gene therapy has been demonstrated to have high levels of efficacy in forming bone in animal models ${ }^{20}$; however, gene therapy may carry considerable clinical risks. ${ }^{21}$ Biomimetic supramolecular nanofibers within collagen scaffolds that sequester/capture endogenously circulating BMPs have been shown to decrease the minimum dose of exogenous BMP-2 required for fusion but do not allow the complete absence of exogenous BMP-2. ${ }^{22}$

To date, cell-based strategies to improve spinal fusion have focused on mesenchymal stem cells and osteoblast precursors. Importantly, endochondral bone formation is an essential mechanism in spinal fusion. During endochondral ossification, chondrocytes differentiate and hypertrophy, promoting calcification of cartilage before they are subsequently replaced by bone. ${ }^{23}$ Terminally differentiated hypertrophic chondrocytes can also give rise to osteoblasts. $^{24}$

Therefore, the use of endochondral cartilage grafts may be a potential strategy for bone formation. Hypertrophic chondrocytes taken from fracture callus are osteogenic, and stem cell-derived endochondral cartilage stimulates bone healing. ${ }^{25}$ Much less is known about bone formation using chondrocytes from other lineages, such as articular chondrocytes. In contrast to the challenge of securing healthy donor articular chondrocytes as is needed for articular cartilage restoration, osteoarthritic (OA) cartilage is readily salvageable from total joint replacements. We previously demonstrated that endochondral cartilage grafts derived from human OA cartilage can heal segmental long-bone defects in a mouse model. ${ }^{26}$

We performed a pilot study to test the hypothesis that human OA articular chondrocytes could also be recycled and reprogrammed into hypertrophic

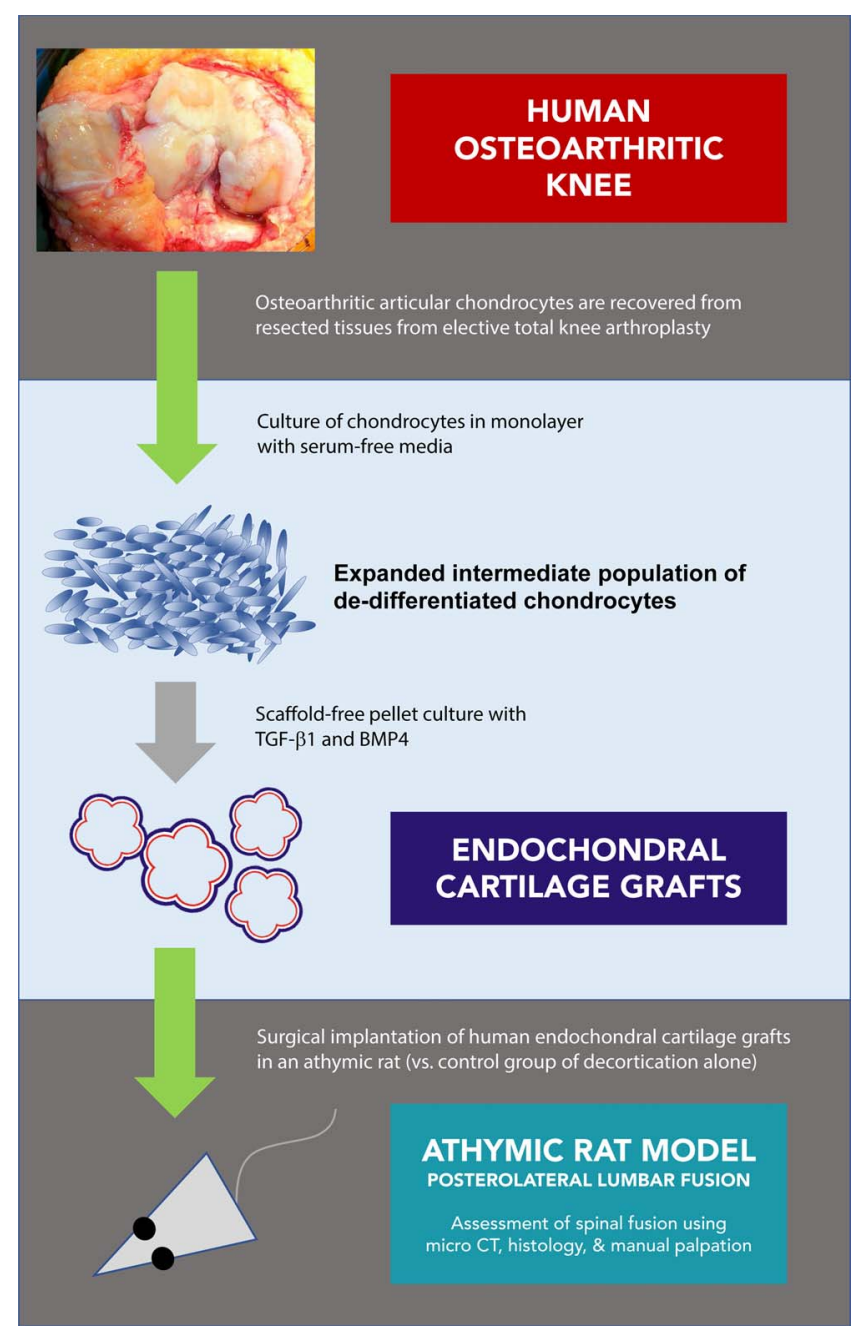

Figure 1. Experimental outline.

chondrocytes capable of achieving posterolateral lumbar fusion in a rat model (Figure 1).

\section{MATERIALS AND METHODS}

Human articular cartilage was isolated from the femoral resections of a 60-year-old man undergoing elective total knee arthroplasty for osteoarthritis. Our Institutional Committee on Human Research reviewed this protocol and ruled that it did not constitute human subject research because patient identifiers and other protected health information were not collected. Articular chondrocytes were isolated with collagenase digestion as previously described. ${ }^{26}$ Cells were expanded in monolayer to passage 6 ( 700-fold expansion of cell numbers) using MesenGro MSC media (StemRD, Burlingame, California) containing Gibco GlutaMAX supplement (Life Technologies, Grand Island, New York). Expansion in monolayer allowed the artic- 
ular chondrocytes to lose their terminally differentiated phenotype.

\section{Endochondral Cartilage Grafts}

Passaged chondrocytes were released from monolayer with trypsin and centrifuged for 5 minutes at $500 \mathrm{~g}$. The resultant cell pellets were cultured for 4 weeks in insulin-transferrin-selenium (ITS) media (DMEM/F-12, ITS premix, Becton Dickinson, Franklin Lakes, NJ; $50 \mathrm{mg} / \mathrm{mL}$ ascorbic acid; $0.1 \%$ bovine serum albumin; $100 \mathrm{mg} / \mathrm{mL}$ sodium pyruvate; and $25 \mathrm{mM}$ HEPES) containing TGF- $\beta 1$ $(10 \mathrm{ng} / \mathrm{mL})$ and BMP4 (200 ng/mL).

The resultant cartilage grafts were implanted into rats as described below.

\section{Surgical Procedure}

All animal protocols were reviewed and approved by our Institutional Animal Care and Use Committee. Eight- to 12-week-old, male athymic nude rats (strain Hsd:RH-Foxn1 ${ }^{\text {rnu }}$, Harlan Sprague Dawley Inc, Indianapolis, Indiana) were used. A standardized rat model of single-level posterolateral lumbar spinal fusion was used. ${ }^{27}$ Animals were placed under isoflurane anesthesia and positioned prone. The lumbar region of the dorsal skin was shaved and prepped with povidone-iodine. Buprenorphine and meloxicam were injected subcutaneously. A 20-mm midline skin incision was made, and the paravertebral muscles and the most posterior aspects of the spinous processes were exposed. The paravertebral muscles were reflected laterally to expose the spinous processes and lamina bilaterally. The facet joints and transverse processes of 2 adjacent lumbar vertebrae were exposed. The transverse processes, lamina, and spinous processes were decorticated. In experimental animals $(n=5)$, endochondral cartilage grafts were laid down bilaterally in direct contact with the decorticated posterior elements (approximately $25-30 \mathrm{~mm}^{3}$ of graft on each side). In control animals $(n=5)$, decortication alone was performed and no grafts were implanted. The wound was closed in layers. Postoperatively, animals were allowed activity without restriction.

\section{Determination of Fusion}

A SCANCO (Wayne, Pennsylvania) Medical VivaCT 40 Preclinical Scanner was used for in vivo scans. Rats were anesthetized using inhaled isoflur- ane and placed in the supine position in the scanner. The spine was scanned from the $\mathrm{L} 1$ to $\mathrm{S} 1$ vertebrae at a thickness of $10 \mu \mathrm{m}$. A SCANCO Medical $\mu \mathrm{CT}$ 50 Specimen Scanner was used for ex vivo scans. In vivo micro-computed tomography (micro-CT) of the lumbar spine was performed under isoflurane anesthesia on postoperative day 0 or 1 , and it was repeated again 4 weeks after surgery. Animals were killed 8 weeks after implantation. The lumbar spines and sacrum were harvested en bloc with retention of the surrounding musculature.

Manual palpation of the specimen prior to fixation was performed by 3 independent observers blinded to treatment groups. Each observer was asked to rate the surgical level as either fused or not fused. The observers had no knowledge of any imaging studies.

A high-resolution ex vivo micro-CT of the lumbar spine was then performed. Dissected specimens containing the lumbar spine and surrounding musculature were placed in $70 \%$ ethanol and then scanned at a thickness of $10 \mu \mathrm{m}$. Intervertebral fusion as assessed by micro-CT was defined as the presence of bone that bridged and was in continuity with the native bony elements of the 2 operated adjacent vertebrae. Cross-sectional micro-CT images of the spine encompassing the operated levels were reviewed, and regions of interest (ROIs) that included new fusion mass were outlined. The native bone of the vertebrae was excluded from the ROI. Manual definition of ROIs was performed on all sections that displayed a distinct bony morphology. The "morph" functionality of the SCANCO image analysis software was used to interpolate the contour of the fusion mass for all cross sections between the manually analyzed images. Each automated ROI was evaluated for accuracy and, if necessary, manually adjusted. This cross-sectional information was then used to calculate the total volume of the fusion mass. All micro-CT analysis and ROI contouring were performed by an investigator blinded to treatment groups.

The volumes of residual endochondral cartilage grafts in each fusion mass at 4 and 8 weeks after surgery were estimated by evaluation of coronal micro-CT images. Remnant grafts were identified as spherical, nonmineralized regions within the mineralized tissue of the fusion masses. All sections containing an individual graft were reviewed, the largest diameter of the graft was measured, and the 

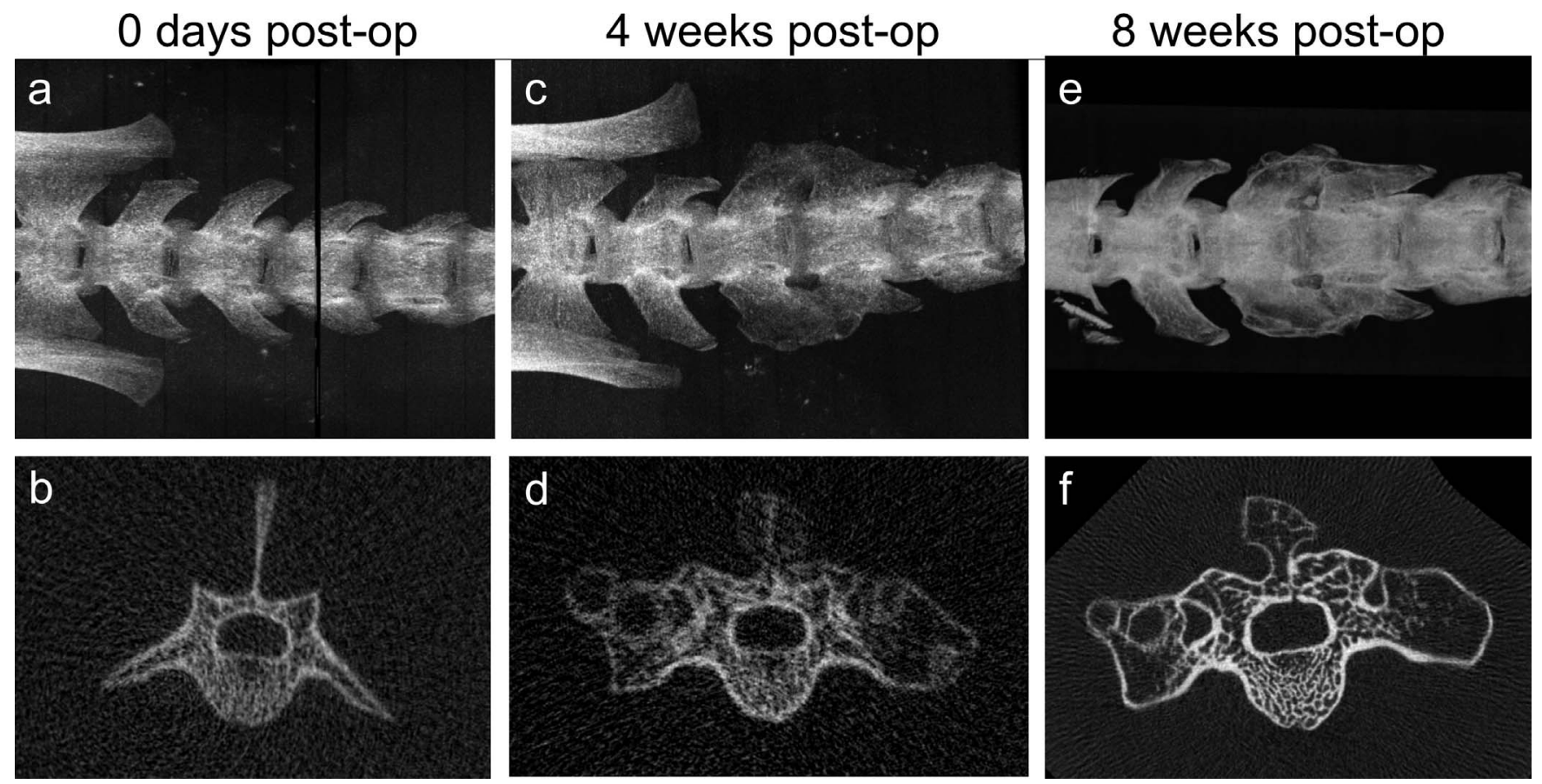

Figure 2. Micro-computed tomography images showing time course of single-level posterolateral lumbar spinal fusion using hypertrophic chondrocyte pellet grafts in a single animal. (a, b) In vivo immediately after implantation. (c, d) In vivo 4 weeks postoperatively. (e, f) Ex vivo 8 weeks postoperatively.

radius $(r)$ of the graft was calculated. The volume of each graft was estimated to be $4 / 3 \pi r^{3}$.

After imaging, samples were then decalcified with trichloroacetic acid, fixed with paraformaldehyde, embedded in paraffin, and sectioned. Adjacent sections were stained with safranin-O/fast green and Masson trichrome. ${ }^{26}$

Two-tailed Fisher exact tests were used for categoric data analysis, and Student $t$-tests were used for analysis of continuous variables. SiegelCastellan fixed-marginal multirater $\kappa$ was used to assess interrater agreement for manual palpation. Significance was set at $P<.05$.

\section{RESULTS \\ Radiographic Fusion}

There was no evidence of new bone formation or intervertebral fusion in the control group. In the animals receiving cartilage grafts, there was new bone in all 5 animals. At 8 weeks postoperatively, bilateral fusion was identified on micro-CT in 4 animals, and unilateral fusion was identified in the remaining animal (Figure 2). Fusion could also be seen on micro-CT at 4 weeks postoperatively. Bilateral fusion was statistically significantly increased in the group receiving cartilage grafts $(P=$ .047). With relaxed criteria of unilateral fusion, the
$P$ value is .007 . When assessed as 10 independent fusion sites the $P$ value was .0001 .

Assessment of the experimental animals through time showed that there was no mineralization (as expected) at time zero. Mineralization and new bone formation were present in the micro-CT images obtained at 4 weeks postoperatively. Spherical unmineralized areas were found in fusion masses at 4 and 8 weeks. The size, location, and appearance of the corresponding spherical regions were consistent with those of endochondral cartilage grafts that had not been completely remodeled into bone (Figure 3).

Three end points for maturation of the fusion masses from 4 to 8 weeks were assessed: (1) volume of bone in the fusion masses, (2) volume of remnant cartilage grafts in the fusion masses, and (3) mean bone mineral density of the fusion masses (Figure 4).

There was a trend toward increased volume of bone in the fusion masses from 4 to 8 weeks; however, this difference did not reach statistical significance (mean volume of $52 \mathrm{~mm}^{3}$ versus 63 $\mathrm{mm}^{3} ; P=.32$ ). The mean volume of cartilage grafts in each animal decreased significantly from $25 \mathrm{~mm}^{3}$ at 4 weeks to $8 \mathrm{~mm}^{3}$ at 8 weeks $(P=.006)$. A total of 40 endochondral spherical grafts were identified in the in vivo micro-CT images obtained at 4 weeks postoperatively. At 8 weeks, 13 of these grafts had 

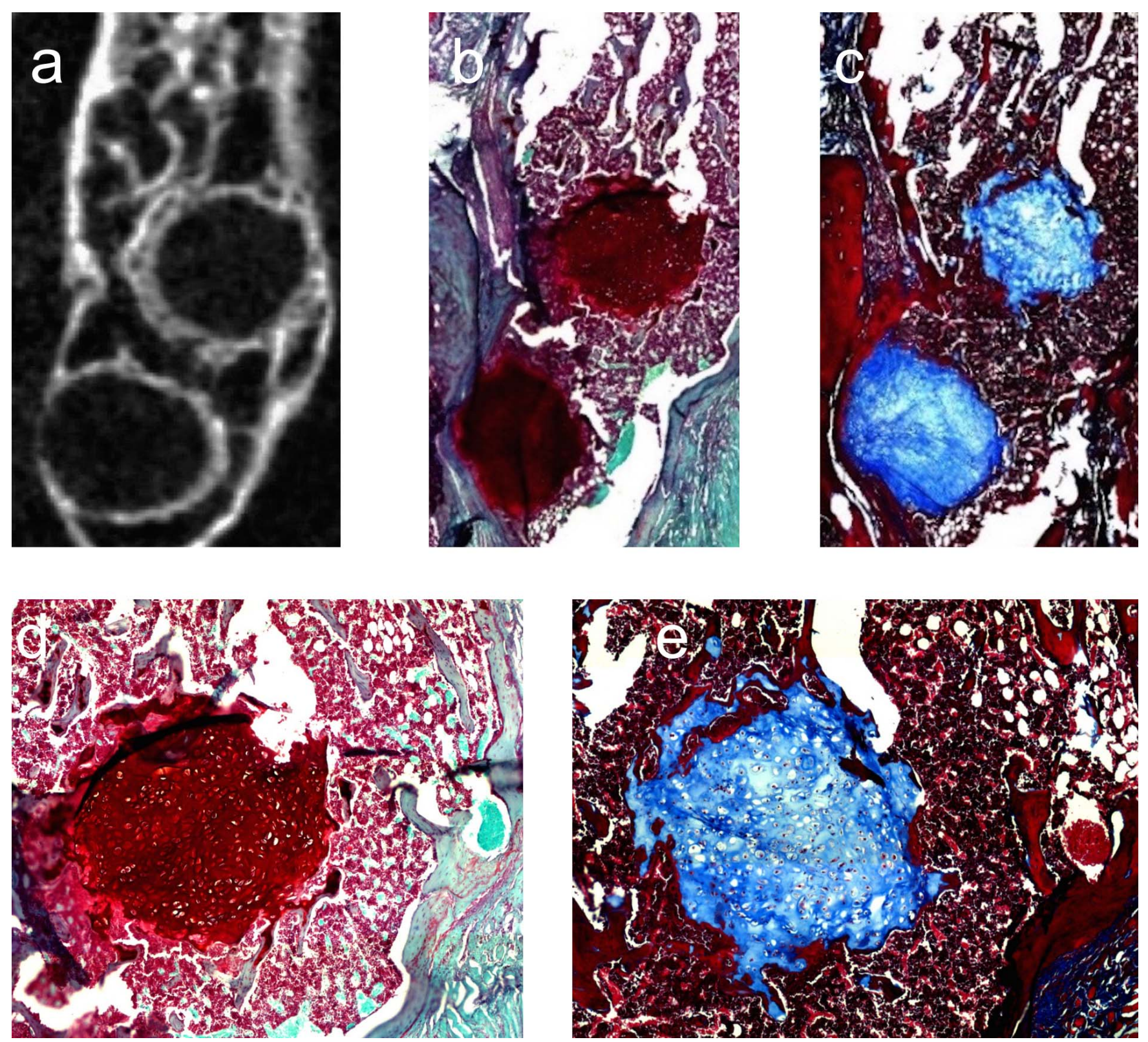

Figure 3. Radiographic and histologic appearance of remnant cartilage grafts at 8 weeks. Micro-computed tomography (a), safranin-O staining (b, d), and Masson trichrome staining (c, e) of fusion masses show spherical areas of unmineralized cartilaginous tissue.

completely remodeled into mineralized tissue, and the volume of all the remaining grafts had decreased. Mean bone mineral density increased from $719 \mathrm{mg}$ hydroxyapatite $/ \mathrm{cm}^{3}$ at 4 weeks to 954 $\mathrm{mg}$ hydroxyapatite $/ \mathrm{cm}^{3}$ at 8 weeks $(P=.003)$.

\section{Manual Palpation}

Manual palpation was performed on all spine specimens after harvest at 8 weeks by 3 independent observers blinded to treatment. No control animal was judged to be fused except for 1 animal that was judged to be fused by 1 observer. In contrast, all experimental animals were judged to be fused except for 1 animal that was judged to be unfused by 1 observer. Therefore, controls were determined to be fused in 1 of 15 assessments, and experimental specimens were determined to be fused in 14 of 15 assessments. This difference was statistically significant $(P=.0001)$. The Siegel-Castellan fixedmarginal multirater $\kappa$ for interrater agreement was 0.73. A summary of results is presented in the Table.

An unblinded examination of the specimens and imaging was performed. No characteristics were identified that explained why 1 examiner thought the control animal was fused; however, the exper- 
a

Volume of bone in fusion mass

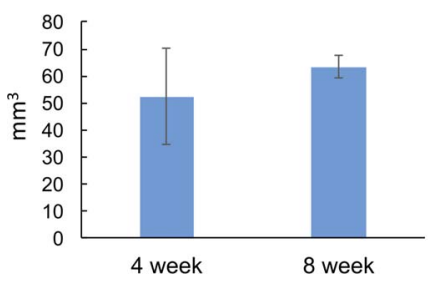

b Volume of cartilage graft in fusion mass

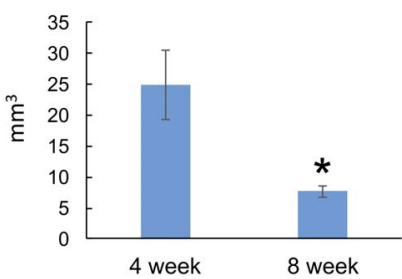

C Mean bone mineral

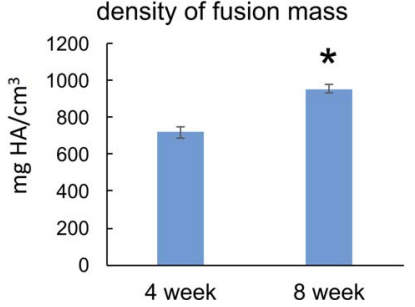

Figure 4. Maturation of fusion masses. From 4 to 8 weeks, the amount of cartilage in the fusion masses decreased and mean bone mineral density increased. There was a trend toward increased bone volume with time; however, this did not reach statistical significance.

imental animal that was judged to be incompletely fused was the animal with unilateral fusion.

An unblinded examination of the specimens and imaging was performed after manual palpation was complete. No characteristics were identified that might have explained why 1 examiner judged the control animal to be fused; however, the experimental animal that was judged to be incompletely fused was found to be the only animal with radiographic unilateral fusion.

\section{Histologic Assessment}

Histology was performed to evaluate the tissue composition of the fusion masses. They consisted of shells of cortical bone with an interior of woven bone interspersed with marrow elements. The spherical, unmineralized areas of tissue seen on micro-CT cross sections had a histologic appearance consistent with unremodeled endochondral cartilage grafts (Figure 3). Relative to the complete fusion mass, however, the persistent cartilage component represented a small portion of the volume (Figure 4).

\section{DISCUSSION}

Bone marrow-derived and adipose-derived stem cells have been used successfully to induce bone formation in a variety of preclinical models. ${ }^{28-31}$ However, this is the first study to use OA articular chondrocytes as bone graft in a posterolateral spinal fusion model. Use of chondrocytes harvested from OA cartilage that would otherwise be discarded is an attractive allograft option given the relative plethora of supply and absence of added morbidity to donors beyond the electively performed total knee arthroplasty.

The results of this study show the osteopromotive (and likely osteogenic) activity that can be achieved with cartilage rather than osteoblasts. Our control group, which only underwent decortication, showed zero fusion and zero new bone, in contrast to the $100 \%$ success rate of the experimental group, defined by unilateral or bilateral fusion. In this study, cartilage had not completely transformed to bone by 8 weeks. It is possible that these cartilage structures will remodel over time. Nevertheless, bridging bone was achieved in all experimental animals.

Because our goal for this study was to assess the feasibility of using OA cartilage as a bone graft substitute in posterolateral spinal fusion, the control group selected comprised animals treated with decortication alone. This was done to verify that the surgical approach itself would not generate heterotopic bone. A third group receiving iliac crest autograft or rhBMP-2 may have provided a performance comparison between cartilage grafts and a gold standard; however, this topic is beyond the scope of this study, and optimization of the pellet size and quantity of cartilage grafts has not yet been completed.

Comparisons to fusion rates obtained with other bone graft substitutes should be interpreted with caution. Nevertheless, compared with data from other experiments using the rat model of single-level lumbar posterolateral fusion, the performance of these cartilage grafts is promising. Hsu et al. ${ }^{30}$

Table. Spinal fusion evaluated by radiography and manual palpation by 3 blinded examiners.

\begin{tabular}{|c|c|c|c|c|c|}
\hline & \multicolumn{2}{|c|}{ Radiographic Fusion, \% } & \multicolumn{3}{|c|}{ Manual Palpation, \% } \\
\hline & Unilateral or Bilateral & Bilateral & Examiner 1 & Examiner 2 & Examiner 3 \\
\hline Control $(\mathrm{n}=5)$ & 0 & 0 & 20 & 0 & 0 \\
\hline Graft $(\mathrm{n}=5)$ & 100 & 80 & 80 & 100 & 100 \\
\hline$P$ value & .007 & .047 & & $.0001(\kappa=0.7$ & \\
\hline
\end{tabular}


reported that $1 \mu \mathrm{g}$ of rhBMP-2 (low dose) had a $50 \%$ unilateral fusion rate and $0 \%$ bilateral fusion $(\mathrm{n}=8)$ at 4 weeks. Animals treated with $10 \mu \mathrm{g}$ of rhBMP-2 (high dose) had a 100\% bilateral fusion rate. Grauer et al. ${ }^{32}$ reported a $30 \%$ fusion rate for athymic rats treated with bone autograft after 6 weeks $(\mathrm{n}=20)$. Bomback et al. ${ }^{33}$ reported a $39 \%$ fusion rate at 6 weeks with Grafton DBM $2 \mathrm{~cm}^{3} / \mathrm{kg}$ $(\mathrm{n}=30)$. All animals treated with $560 \mu \mathrm{g}$ of osteogenic protein-1 fused at 3 weeks. Our study cohort had $100 \%$ fusion (unilateral fusion criteria) and $80 \%$ bilateral fusion $(n=5)$ with cartilage grafts at 4 weeks.

This study establishes a proof of concept that OA cartilage salvaged from surgical waste generated from routine orthopaedic arthroplasty surgery can be processed in a manner to generate a bone graft substitute that is effective, and likely comparable if not superior to low-dose rhBMP-2.

\section{REFERENCES}

1. Bridwell KH, Sedgewick TA, O'Brien MF, Lenke LG, Baldus $C$. The role of fusion and instrumentation in the treatment of degenerative spondylolisthesis with spinal stenosis. J Spinal Disord. 1993;6(6):461-472.

2. Dickson JH, Mirkovic S, Noble PC, Nalty T, Erwin WD. Results of operative treatment of idiopathic scoliosis in adults. $J$ Bone Joint Surg Am. 1995;77(4):513-523.

3. West JL 3rd, Bradford DS, Ogilvie JW. Results of spinal arthrodesis with pedicle screw-plate fixation. J Bone Joint Surg Am. 1991;73(8):1179-1184.

4. Yadla S, Maltenfort MG, Ratliff JK, Harrop JS. Adult scoliosis surgery outcomes: a systematic review. Neurosurg Focus. 2010;28(3):E3.

5. Zdeblick TA. A prospective, randomized study of lumbar fusion: preliminary results. Spine (Phila Pa 1976). 1993;18(8):983-991.

6. Sengupta DK, Truumees E, Patel CK, et al. Outcome of local bone versus autogenous iliac crest bone graft in the instrumented posterolateral fusion of the lumbar spine. Spine (Phila Pa 1976). 2006;31(9):985-991.

7. Miura Y, Imagama S, Yoda M, Mitsuguchi H, Kachi H. Is local bone viable as a source of bone graft in posterior lumbar interbody fusion? Spine (Phila Pa 1976). 2003;28(20):2386-2389.

8. Rajaee SS, Bae HW, Kanim LE, Delamarter RB. Spinal fusion in the United States: analysis of trends from 1998 to 2008. Spine (Phila Pa 1976). 2012;37(1):67-76.

9. Parker S, Shau D, Mendenhall S, et al. Cost per qualityadjusted life year gained of revision lumbar surgery for adjacent segment disease, pseudoarthrosis, and same-level recurrent stenosis: defining the value of surgical intervention. Presented at: 28th Annual Meeting of the AANS/CNS Section on Disorders of the Spine and Peripheral Nerves; March 7-10, 2012; Orlando, FL.

10. Eichholz KM, Ryken TC. Complications of revision spinal surgery. Neurosurg Focus. 2003;15(3):E1.
11. Guerin P, El Fegoun AB, Obeid I, et al. Incidental durotomy during spine surgery: incidence, management and complications: a retrospective review. Injury. 2012;43(4):397401.

12. Francis CS, Mobin SS, Lypka MA, Rommer E, Yen S, Urata MM, et al. rhBMP-2 with a demineralized bone matrix scaffold versus autologous iliac crest bone graft for alveolar cleft reconstruction. Plast Reconstr Surg. 2013;131(5):11071115.

13. Kim HJ, Buchowski JM, Zebala LP, Dickson DD, Koester L, Bridwell KH. RhBMP-2 is superior to iliac crest bone graft for long fusions to the sacrum in adult spinal deformity: 4- to 14-year follow-up. Spine (Phila Pa 1976). 2013;38(14):1209-1215.

14. Carreon LY, Glassman SD, Djurasovic M, et al. RhBMP-2 versus iliac crest bone graft for lumbar spine fusion in patients over 60 years of age: a cost-utility study. Spine (Phila Pa 1976). 2009;34(3):238-243.

15. Carragee EJ, Hurwitz EL, Weiner BK. A critical review of recombinant human bone morphogenetic protein-2 trials in spinal surgery: emerging safety concerns and lessons learned. Spine J. 2011;11(6):471-491.

16. US Food and Drug Administration. FDA public health notification: life-threatening complications associated with recombinant human bone morphogenetic protein in cervical spine fusion. Issued July 1, 2008. http://web.archive.org/web/ 20170118090844/http://www.fda.gov/MedicalDevices/Safety/ AlertsandNotices/PublicHealthNotifications/ucm062000.htm. Accessed April 10, 2013.

17. Wong DA, Kumar A, Jatana S, Ghiselli G, Wong K. Neurologic impairment from ectopic bone in the lumbar canal: a potential complication of off-label PLIF/TLIF use of bone morphogenetic protein-2 (BMP-2). Spine J. 2008;8(6):10111018.

18. Epstein NE. Complications due to the use of BMP/ INFUSE in spine surgery: the evidence continues to mount. Surg Neurol Int. 2013;4(suppl 5):S343-S352.

19. Rihn JA, Kirkpatrick K, Albert TJ. Graft options in posterolateral and posterior interbody lumbar fusion. Spine (Phila Pa 1976). 2010;35(17):1629-1639.

20. Baltzer AW, Lieberman JR. Regional gene therapy to enhance bone repair. Gene Ther. 2004;11(4):344-350.

21. Manilla P, Rebello $\mathrm{T}$, Afable $\mathrm{C}$, et al. Regulatory considerations for novel gene therapy products: a review of the process leading to the first clinical lentiviral vector. Hum Gene Ther. 2005;16(1):17-25.

22. Lee SS, Huang BJ, Kaltz SR, et al. Bone regeneration with low dose BMP-2 amplified by biomimetic supramolecular nanofibers within collagen scaffolds. Biomaterials. 2013;34(2):452-459.

23. Gerstenfeld LC, Shapiro FD. Expression of bonespecific genes by hypertrophic chondrocytes: implication of the complex functions of the hypertrophic chondrocyte during endochondral bone development. J Cell Biochem. 1996;62(1):19.

24. Yang L, Tsang KY, Tang HC, Chan D, Cheah KSE. Hypertrophic chondrocytes can become osteoblasts and osteocytes in endochondral bone formation. Proc Natl Acad Sci U S A. 2014;111(33):12097-12102.

25. Bahney CS, Hu DP, Taylor AJ, et al. Stem cell-derived endochondral cartilage stimulates bone healing by tissue transformation. J Bone Miner Res. 2014;29(5):1269-1282. 
26. Bahney CS, Jacobs L, Tamai R, et al. Promoting endochondral bone repair using human osteoarthritic articular chondrocytes. Tissue Eng Part A. 2016;22(5-6):427-435.

27. Grauer JN, Bomback DA, Lugo R, Troiano NW, Patel TC, Friedlaender GE. Posterolateral lumbar fusions in athymic rats: characterization of a model. Spine J. 2004;4(3):281-286.

28. Wang JC, Kanim LE, Yoo S, Campbell PA, Berk AJ, Lieberman JR. Effect of regional gene therapy with bone morphogenetic protein-2-producing bone marrow cells on spinal fusion in rats. J Bone Joint Surg Am. 2003;85A(5):905911.

29. Viggeswarapu M, Boden SD, Liu Y, et al. Adenoviral delivery of LIM mineralization protein-1 induces new-bone formation in vitro and in vivo. J Bone Joint Surg Am. 2001;83A(3):364-376.

30. Hsu WK, Wang JC, Liu NQ, et al. Stem cells from human fat as cellular delivery vehicles in an athymic rat posterolateral spine fusion model. J Bone Joint Surg Am. 2008;90(5):1043-1052.

31. Lopez MJ, McIntosh KR, Spencer ND, et al. Acceleration of spinal fusion using syngeneic and allogeneic adult adipose derived stem cells in a rat model. J Orthop Res. 2009;27(3):366-373.

32. Grauer JN, Bomback DA, Lugo R, Troiano NW, Patel TC, Friedlaender GE. Posterolateral lumbar fusions in athymic rats: characterization of a model. Spine J. 2004;4(3):281-286.
33. Bomback DA, Grauer JN, Lugo R, Troiano N, Patel T, Friedlaender GE. Comparison of posterolateral lumbar fusion rates of Grafton Putty and OP-1 Putty in an athymic rat model. Spine (Phila Pa 1976). 2004;29(15):1612-1617.

Disclosures and COI: The authors received no funding for this study and report no conflicts of interest. This project was funded by a University of California, San Francisco, School of Medicine Pilot Grant for Junior Investigators in Basic and Clinical/ Translational Sciences.

Corresponding Author: Alfred C Kuo, MD, PhD, San Francisco VA Medical Center, 4150 Clement Street Box 112, San Francisco, CA 94121. Phone: (415) 221-4810; Email: ackuo1@yahoo.com.

Published 21 December 2018

This manuscript is generously published free of charge by ISASS, the International Society for the Advancement of Spine Surgery. Copyright (C) 2018 ISASS. To see more or order reprints or permissions, see http://ijssurgery.com. 